\title{
EXPLORING EXPRESSION OF FORM, ACTION AND INTERACTION
}

\author{
Steven Kyffin, Loe Feijs, and Tom Djajadiningrat \\ Steven Kyffin, Philips Design, Eindhoven, Netherlands, Loe Feijs, and Tom Djajadiningrat, \\ Eindhoven University of Technology, Netherlands
}

\begin{abstract}
The central theme in Ambient Intelligence is that powerful computation, communication and storage facilities are available, but are invisible. We believe that objects will play an important role as mediators. In order to make models of mediation and to study the mediation of behaviour, traditional modelling techniques, such as foam models, rendering etc. fall short. Although it is easy to make two-dimensional models of screens and front-panels and connect actions to the corresponding buttons, this approach is inadequate for new types of interfaces and behaviours which are not screen-based. Therefore we have experimented with an approach which we call $4 \mathrm{D}$ Sketching. The term 4D refers to the fact that it includes sketching in 3D space with the active behaviour adding a fourth, temporal dimension. We use foam, cardboard, a glue-gun, and other easy-to-use materials for the spatial sketching. We use a microprocessor, servo-motors and sensors to sketch active behaviour. The article describes the underlying philosophy, the technical aspects and a number of experiences gathered so-far, including student work from a project called Semotion.
\end{abstract}

Key words: Emotions, sketching, model making, software, product semantics, Movement, Motion, Dynamic behaviour, Robotics.

\section{INTRODUCTION}

The vision of ambient intelligence as put forward by Weiser and adopted by ISTAG and many companies, forms the basis of considerable R\&D efforts. These efforts will affect and shape the future of home information systems and eventually the homes themselves and the ways of being of people at 
home. Examples such as the GeorgiaTech's aware home, the Philips Home Lab in Eindhoven, the inHaus in Duisburg, the OpenHomeAsia project in Singapore, the Equator project in the UK are carriers to explore ambient intelligence. The central theme is that powerful computation, communication and storage facilities are available, but are invisible. In Marzano's "La Casa Prossima Futura" the black boxes have disappeared and the living room contains objects and furniture, again. Then if the traditional terminals disappear, what are the mediators between people and this hidden intelligence? How do people control, and get feedback from, these resources in a way that is meaningful and even attractive at a human level (not just efficient at a machine level).

We believe that objects will play an important role as mediators, (besides automatic detectors and wall-mounted displays). In the simplest cases, ordinary objects get tagged or equipped with buttons and displays. But to take full advantage of the richness of human-object interaction and to use the potential of affective (emotional) interactions, there is a need for a new approach. This is an important aspect of home information systems that deserves more attention, also by industrial designers. At present, there is an emphasis on the input rather than the output side: sensor technology, video recognition and object tracking. Also, in research into affective computing and products there is emphasis on the recognition of emotions rather than affect expression e.g. Picard at MIT and Wensveen at TU/e. Most often the output is restricted to flat displays (or, perhaps in Japan, humanoid or animal-inspired robots). An interesting exception is the key table and picture frame developed in the Equator project. Although many traditional home products such as toasters and coffee cans, which do have mechanically moving parts, follow a trend to converge with computing, this convergence comes in the form of electronic displays and buttons being added to traditional forms. As an alternative we explore adding behavioral expression to the existing movement possibilities.

We envisage the need for new types of processes and tools to support the creation of the envisaged new product types. In our work we focus on product behaviour that is enriched with physical movements. Several possibilities exist: either the product is moving anyhow, or the movements are added just for the sake of communication. In both cases, the designer has considerable freedom how to shape the movements and the interactions.

Forms, either concrete or abstract, always carry meanings. It is the responsibility of the designer to make good use of these meanings, for example, to make products beautiful, to stress the importance of certain values, or to improve a product's ease of use and to promote or negotiate enriched experiences between people (communities) and people, people and objects and in time between objects (systems of objects) and objects. Design 
uses its own languages for this purpose, just as poets, painters, journalists, sculptures, film makes and so on do. The topic of this paper is how to explore the combined usage of form, colour and behaviour as a design language. We have to look for new ways of exploring behaviour, not separately, but in relation to traditional forms. The 4D sketching approach is helpful to find out in how far object behaviour can be used as a language and in how far it can communicate meaning.

Let us define sketching as the creation of form proposals that are:

- fast

- ambiguous

A sketch is fast in order to allow the designer to evaluate the proposal and let others evaluate the proposal while still having time for modifications and new proposals. A sketch is ambiguous if it does not suggest a higher level of precision than intended by the designer in a certain early stage of the development process. Ambiguity prevents an evaluator from focusing on details that are not supposed to be fixed yet. In other words: sketching can be used to explore a language of forms and their meanings before making the real decisions.

In this paper we show how sketching can be extended from 2- and 3dimensional sketching to include behavioural aspects too.

\section{TOWARDS RICHER MODELLING}

New technologies have led to a redefinition of the concept of 'object'. Those objects, which exist only inside the computer, are referred to as 'virtual', and for the time being it is still impossible to give them a physical presence, because there are not yet any materials that conform to their forms. However, these objects do live within the realms of another physicality, a host, so to speak. It is the seamless integration or convergence of the two levels of existence that design needs to begin to reconcile. One of the 'raw materials' the designer will be using in the future consists of software technology.

Whereas the 'old' designer would use 3D forms, colours and materials, we envisage a need for designers who can use communication and software since these are the 'raw materials' for the design of the virtual. Designers and students of design continue to use a multitude of different media to 'draw' out, illustrate and explore their design ideas. Two dimensional, representational techniques, using paper, pens and inks, have often proved sufficient for representing intended ideas. The introduction of computerbased mediums has accelerated and brought realistic accuracy to the process. 
The computer has also given rise to the integration of the two and threedimensional aspects of the 'formal' aspects.

In the past designers would typically draw ideas on paper and explore form, mass, scale and weight based issues in the third dimension, eventually bringing the two aspects together in a single object as an appearance model. Today the computer enables both issues to be tackled simultaneously with the added advantage that through rapid prototyping techniques, such as stereo-lithography and five-axis milling, a 'hard' representation can be prepared with ease. The geometry data may also be used in the preparation and development of the final production piece without the need to 'rebuild' the form in the computer after the visual and feasibility testing is complete.

However, as designers now face an ever wider spectrum of 'designed' requirements, now including software behaviour, content flow, and even hardware behaviours it is imperative that new forms of drawing or even "sketching' are developed to enable the illustration, discovery and exploration of all these 'conflicting' functional needs in order to ensure the full integration of each effecting the other.

These forms of drawing need be no less 'physical' than those currently used, but probably need to merge both the physical and the 'virtual' elements simultaneously.

Having agreed this premise, the primary issues are: How does one draw this materiality? What are the media of this drawing? How do we combine all the elements without actually making the finished 'thing'? How can we make it fast enough to realise something without actually loosing the idea or inspiration in the realisation process? Will the means be strong enough to encourage others to not only understand the idea but to co-laborate in its 'birth'. Are there means of drawing these new things, which enable others to join in as co-creators?

The examples in Sect 6 will demonstrate that not only have the designers used the form of drawing we call 4D sketching as a means of illustration of a mental model of an idea, but in a very rich way the drawing process has actually released ideas which has enriched and in one case enabled the designer to surpass the mental model altogether.

The three dimensional aspects of the 'drawing' is carried out using 'easy to model' traditional cardboard, foam, paper and small found objects (see Sec. 3.3). The fourth dimension or the animated life drawing is explored using simple electrical, electronic and mechatronic components coupled with the materials from the third dimension (see Sect. 4). Our ambition is to encourage and enable the designers and students of design to draw at all levels from the outset and even to draw as a community rather than as a single person. 


\section{THE TRADITIONAL DESIGN PROCESS: IN HOW FAR DOES IT SUPPORT EXPLORATION OF EXPRESSIVE MOTIONS?}

One of the new challenges in developing the physical and virtual aspects of products simultaneously, is that - as products gain robotic characteristicsautonomous behaviour may no longer be limited to changes in twodimensional displays, but may result in physical, mechanical, threedimensional movements. Traditional industrial design education has taught few, if any, methods or techniques for exploring the expressiveness of such movements. Clearly, expressiveness of appearance has always been an important issue in industrial design. Techniques aimed at exploring appearance-including making moodboards, sketching and pre-modellinghave become established parts of the design process (for an overview see Hummels et al. [4]). Expressiveness of movements, however, is a different matter. Unlike dance or theatre, traditional industrial design has had little need for exploring dynamic expressiveness. We are interested in the semantics of movement, analogous to how in the mid-eighties there was an interest in the semantics of appearance (amongst others Krippendorff \& Butter [5]; Aldersey-Williams et al. [1]). In this section we present a design process that we put together with a view to exploration of dynamic expressiveness. It combines techniques for exploring expressiveness of movement with techniques for exploring expressiveness of appearance:

- collecting video of autonomous mechanical movements

- moodboards

- sketching

- pre-modelling

- acting out (full body movements)

- acting out (hand movements)

- 4D sketching (explorative)

- 4D sketching (presentational)

Below we will discuss the potential role of each technique, and illustrate some techniques by work from two projects. The first project, by Feijs and Kyffin explores the potential of a telephone cradle for mediating the urgency and valence of incoming phone calls. The second project was to raise our awareness of in how far existing design techniques support exploration of dynamic expressiveness. We set up a second year bachelors project at our faculty in which we guided five students in a design process with behavioural expressiveness in mind. In twelve weeks, each student had to design a machine that was to offer its user a walnut, expressing a particular emotion in both its appearance and mechanical movement. The emotions were love, anger, enthusiasm, shyness and panic. In the remainder of this 
section, we provide an overview of some of the more traditional design techniques and our understanding of how these techniques support exploring expressiveness of movement and expressiveness of appearance. The actual 4D sketching technique is discussed in section 4 .

\subsection{Video of Mechanical Movements}

The student project started with collecting video material of a wide variety of mechanical movements, including drawers of $C D$ players/changers, a mechanical gripper at a fancy fair, train doors and an ATM returning a card. As students try to categorize the movement videos they develop a feel for the qualities of movement. For example, $C D$ trays do not move linearly but accelerate and decelerate, cassette compartments turn out to be damped, and $\mathrm{CD}$ changers have compound movements (translation + rotation).

\subsection{Moodboards}

Moodboards are collages of images expressing emotions through form, colour and composition. In the student project, for the most part, the moodboards were useful in exploring expressiveness of appearance in two dimensions, thus providing a stepping stone towards expressiveness of appearance in three dimensions.

\subsection{Sketching}

Sketching proved useful both for expressiveness of appearance and as a first start towards expressiveness of movement (here we mean sketching in two dimensions). For the telephone cradle sketches proved useful to explore the object-character of possible telephone cradles and to discover or reject certain metaphors in an early phase (for example sketches of the same object in active or a sleep-state). Some students sketched cartoon-like storyboards, exploring stances that their product would need to move between.

\section{$3.4 \quad$ Pre-modelling}

Pre-modelling is essentially 3D sketching. Models are made from cardboard and foam (mostly using a glue gun). Pre-models offer a way to explore both expressiveness of appearance and of movement. Some of these pre-models were expressive in a static, sculptural manner, based on the assumption that 
it useful to explore the relation between form and emotions before adding movements (Figure 1 and 2).

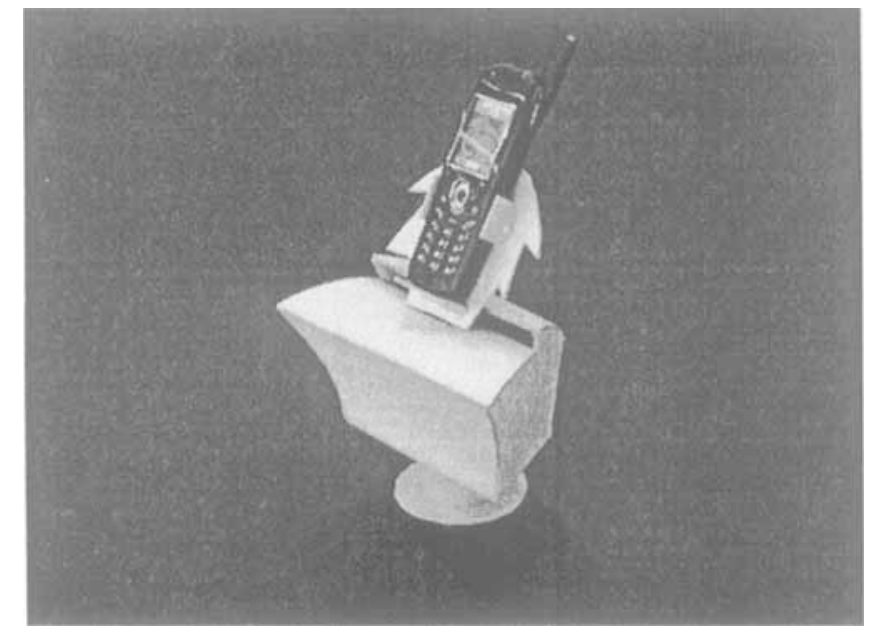

Figure 1. Exploring form and expressed emotions (1).

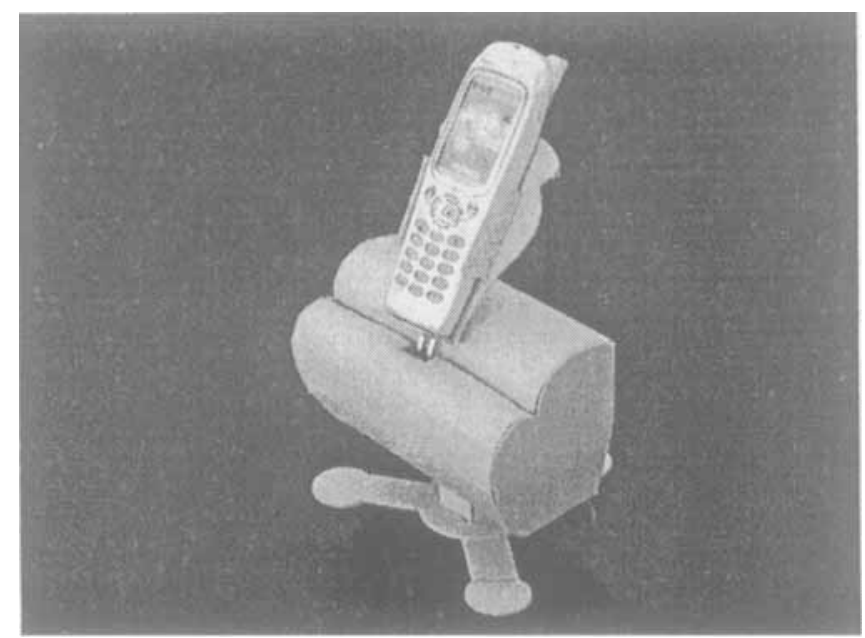

Figure 2. Exploring form and expressed emotions (2).

The cradles of Figures 1 and 2 were intended to convey urgency for negative and positive valences, respectively. Some preliminary investigations revealed that the valence seems to work as intended but the arousal did not (it was reported that the cartoon-like form was associated with "not serious" and "not business-like"). Although no full experiment 
was done yet, we found emotion self-reports using a paper and pencil version of the Self-Assessment Manikin (SAM; Lang 980) useful.

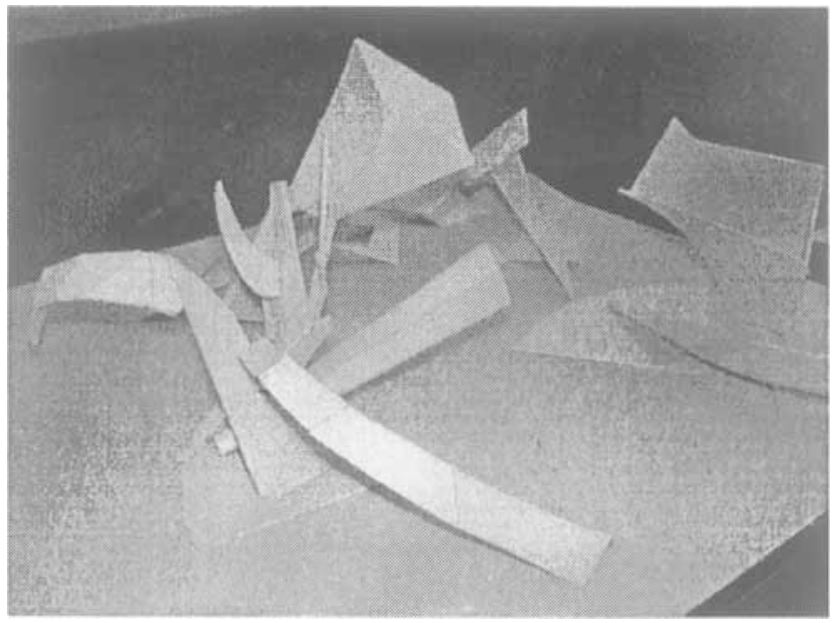

Figure 3. Exploring form and expressed emotions (3).

The pre-models of Figure 3 were used find out whether there was a difference in expressing anger and panic.

Other pre-models already explored qualities of movement. One student added an excentric weight to a hand-sized egg-shaped foammodel, leading to gentle, curvy and soothing movements.

\subsection{Acting out (full body movements)}

We asked students to act out emotions. Video-taping these miniature 'emotional plays' allowed students to analyze and discuss their movements and lent focus and concentration to the session. Acting out is familiar from Burns et al. [3]. In our case, the students acted out not so much the interaction between user, machine and environment but actually stood in for the machine and acted out how it would behave physically. The technique also bears resemblance to that used by Buur et al. [personal communication], though the emphasis is on the emotional content of movements rather than on their interaction style. Initially, we were somewhat hesitant to offer students this technique as we were concerned that this would lead students on an anthropomorphic route, whilst we were aiming for products that had expression appropriate to products. 


\subsection{Acting out (hand movements)}

Somehow the students were aware of this too, and by their own accord they added this technique which is a modification of acting out full-body style. Instead of acting out emotions with their whole bodies, they used only their hands, again recording their movements on video tape. Excluding facial expressions already heightened the level of abstraction.

\subsection{D sketching}

In the next section, we describe a technique developed by the second author which we propose to add to the design process of interactive products to make possible sketching in the temporal dimension. This technique moves beyond on-screen and wizard of oz simulations to physical prototypes with autonomous behaviour. The second author first applied the technique in 'phone robots' of Kyffin and his design and later the technique was explained to our students who used it to further explore the expressiveness of their 'walnut machines'.

\section{SKETCHING IN THE TEMPORAL DIMENSION}

In addition to the techniques of "acting out" at the full-body level and at the hands level, we propose to bring 3D models to life by providing them with actuators, sensors, and processing power. Such augmented cardboard and foam pre-models, could allow designers to explore phyical autonomous movements in a sketchy manner. The actuators, of course, are indispensible to create motion. The sensors are needed to add interactivity or to serve as input when mediating motion. Processing is necessary to control the sequencing and the detailed timing of the actions as well as the rules that connect actuators to sensors. We discuss the actuators first (this section is more technical than the others because the technicalities matter when one must choose among alternative actuators). Although, in principle, there are no limitations to the power and the sophistication of the actuators that could be used, we restrict the discussion here to the types we have been using in at least one of our 4D sketching activities. These are:

- solenoids

- DC motors

- stepping motors

- servo motors

Solenoids are compact actuators that convert electrical energy to linear mechanical action. They contain a coil and a steel plunger that is drawn into 
the coil's magnetic field when energized. They have three disadvantages: their force is not constant over the stroke; most types require return springs, and they tend to get hot when activated for more than a few minutes (we once almost ruined a polystyrene foam model of an active Pacman-like game-support character because its state after the game-session was left undefined).

DC motors are very cheap, small, efficient and silent but an unless a gearbox is fitted, they rotate too fast for most applications (e.g. $3000 \mathrm{rpm}$ ). The torque (rotational force) is inversely proportional to the speed of the output shaft. The gearbox makes them larger and noisier. Controlling their speed is not just a matter of controlling the DC Voltage; a pulse-width modulation is required instead. Controlling their position requires some kind of feedback (which is precisely what a servo-motor does).

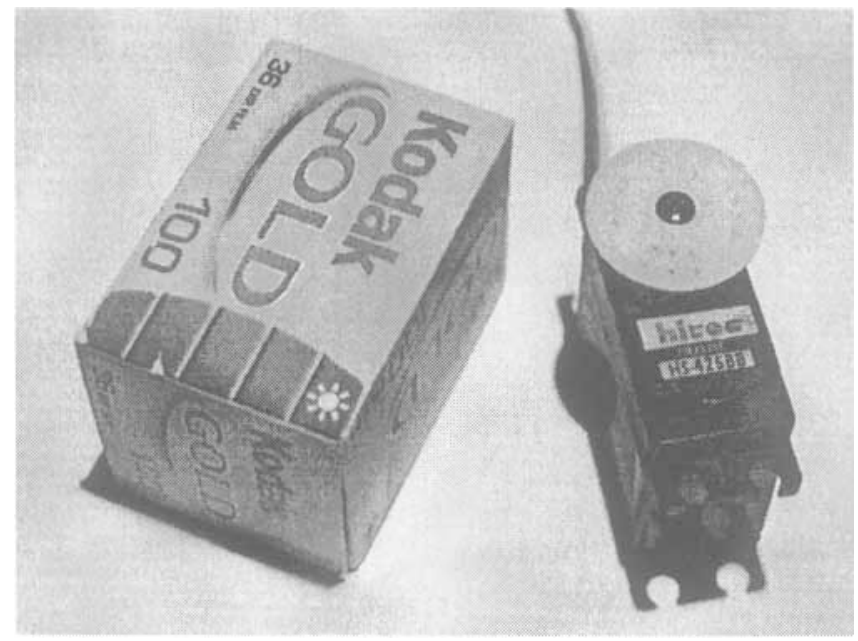

Figure 4. Servo motor

Stepping motors are best understood as electric motors without commutators. The task of the commutators is shifted to the external motor controller, typically the processor plus some extra circuitry. The motor can be held in any fixed position as well as being rotated one way or the other. When a stepping motor is instructed to go to a certain position but the control loop is overtorqued, the motor must be reinitialised and instructed again (this is different for servomotors).

Servo motors contain a DC motor and a variable resistor measuring the rotor's position. These are mechanically and electrically configured to form a feed-back system: the motor will apply more force when encountering resistance and it will try to return to the desired position after a disturbance. 
A small gearbox is built-in. They are not cheap, however (the ones we used cost $40 \$$ each). Their control commands are in the form of repeated pulses whose width indicates the desired position (from 1.25 to 1.75 millisecond). Typically they can turn $210^{\circ}$. This is the preferred actuator for our 4D sketching approach.

Several types of sensors have been used: passive infrared (detecting the heat of humans), active infrared (detecting a reflected signal), and capacitive touch sensors (based on QT310 QProx ${ }^{\mathrm{TM}}$ programmable capacitive IC). These sensors are smaller than the actuators and as such pose no space problems in the sketching. The capacitive touch sensors demand short wires, however; otherwise the wires would act as capacitors themselves (so for a sketch having four touch sensors, a small board with four 8-pin chips had to be housed inside the model).

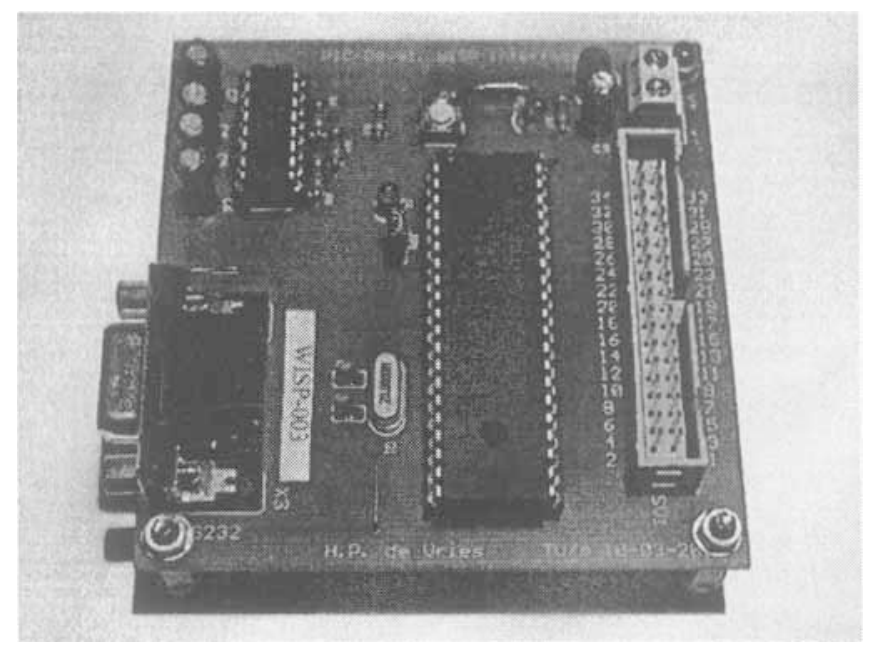

Figure 5. PIC microprocessor board

Processing power is provided by a microprocessor board containing a $20 \mathrm{Mhz}$ PIC processor to be programmed in JAL. (Jet Another Language). Although other processors could be used as well, such as the well-know BASIC stamp, we found PIC convenient because it had been introduced already into our Industrial Design teaching program by our colleagues of the TU/e faculty of Electrical Engineering and also because it has more than 20 configurable input-output pins. The programs can be created using any $\mathrm{PC}$ or laptop computer as the JAL language is open source and cross platform, available on SourceForge. After program compilation and download, the PIC board works in a stand-alone fashion. Downloading is through a protocol called WISP that uses the serial port. Although, in principle, the 
microprocessor board could be housed inside a model, until now we found it convenient to hide it just in a drawer of the demo-table or inside an exhibition console. The processor is reliable and demands no login or boot procedures (it is "turn-key").

The JAL language has Pascal-like programming constructs. It allows direct access to the input-output pins (this is an advantage when compared to higher-level languages such as Java, where the language's security mechanisms implies that hardware is accessed through special interface packages). The data structures are primitive, however: bits and bytes only. To give an impression we show the program which makes a LED connected to pin a0 blink.

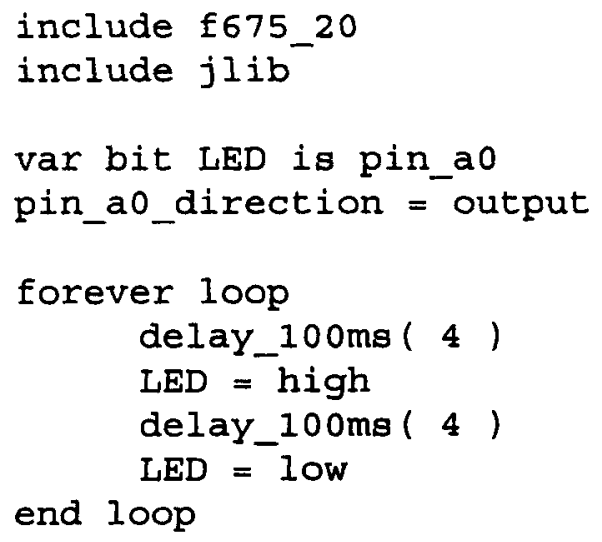

In a typical sketching application with two servo-motors, the program contains two sub-programs which represent finite-state machines, one for each servo motor. Each finite state machine inspects the relevant sensor pins, inspects some variables, makes some decisions, and writes the desired position for its servo-motor to a special variable. There is one main control loop, which invokes both finite state machines. Translating the position value into input output behaviour with delays (in the $1.25-1.75$ millisecond range) for the servo-motors is done in one main loop. The approach resembles the synchronous programming style often used for so-called programming logic controllers (PLCs). The advantage is that the program looks like a set of nicely structured parallel processes, but no multi-tasking operating system is needed. The turn-around time of a typical edit-compilerun cycle is one or two minutes. In our view, this is still fast enough to call the approach "sketching".

During the telephone cradle and walnut vending machine projects we saw variations of $4 \mathrm{D}$ sketching emerge, suited to different phases of the design process. Early on in the process, the servos are combined with quick 
and dirty pre-models. In this phase, the $3 \mathrm{D}$ configuration, the mechanisms and the form details as well as the software, which determines the behaviour, are still open for rapid modifications (Figures 6 and 7). We think of this use of $4 \mathrm{D}$ sketching as explorative. Towards the end of the process, the same technology is embedded in more polished and robust models. In this phase, the 3D configuration and mechanism are already 'frozen': they cannot be easily altered any longer. Of course, some of the subtle details of the behaviour such as timing can still be tweaked. We think of this use of $4 \mathrm{D}$ sketching as presentational.

An analogy can be drawn with $2 \mathrm{D}$ sketching: compared to renderings, rough sketches and presentation sketches are both forms of sketching yet each has its own place in the design process. The former to develop ones ideas on his own or perhaps in a team, the latter to present to managers or clients.

These two phases of 4D sketching are captured in Figure 8. Whilst the explorative version of $4 \mathrm{D}$ sketching allows the designer to carry out both a 'change 3D' and 'change behaviour' loop, with the presentational version the 'change 3D' loop is no longer an option: it is only possible to change the behaviour. Most importantly, the 4D sketching method offers a smooth transition from 'explorative' to 'presentational': the components and workflow remain the same.

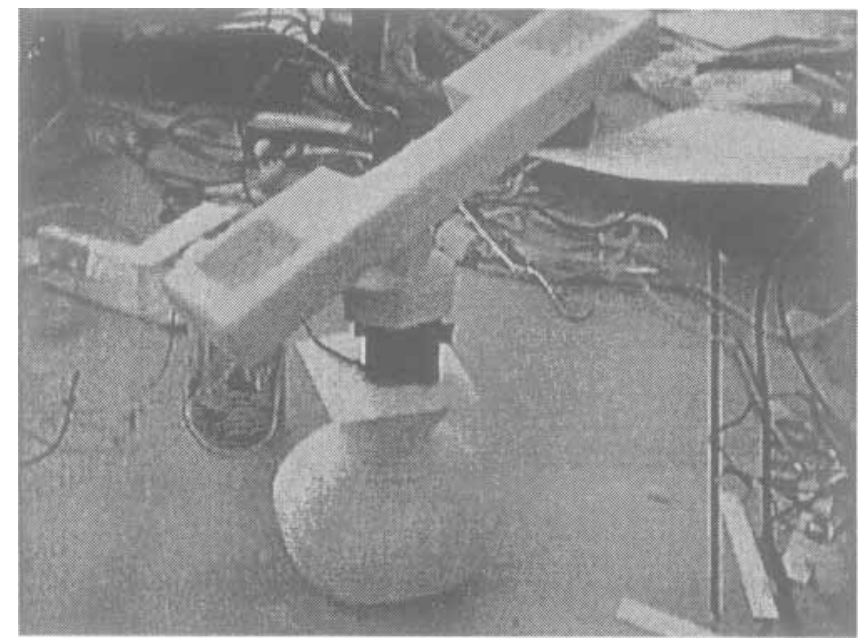

Figure 6. 4D sketching explorative(1) 


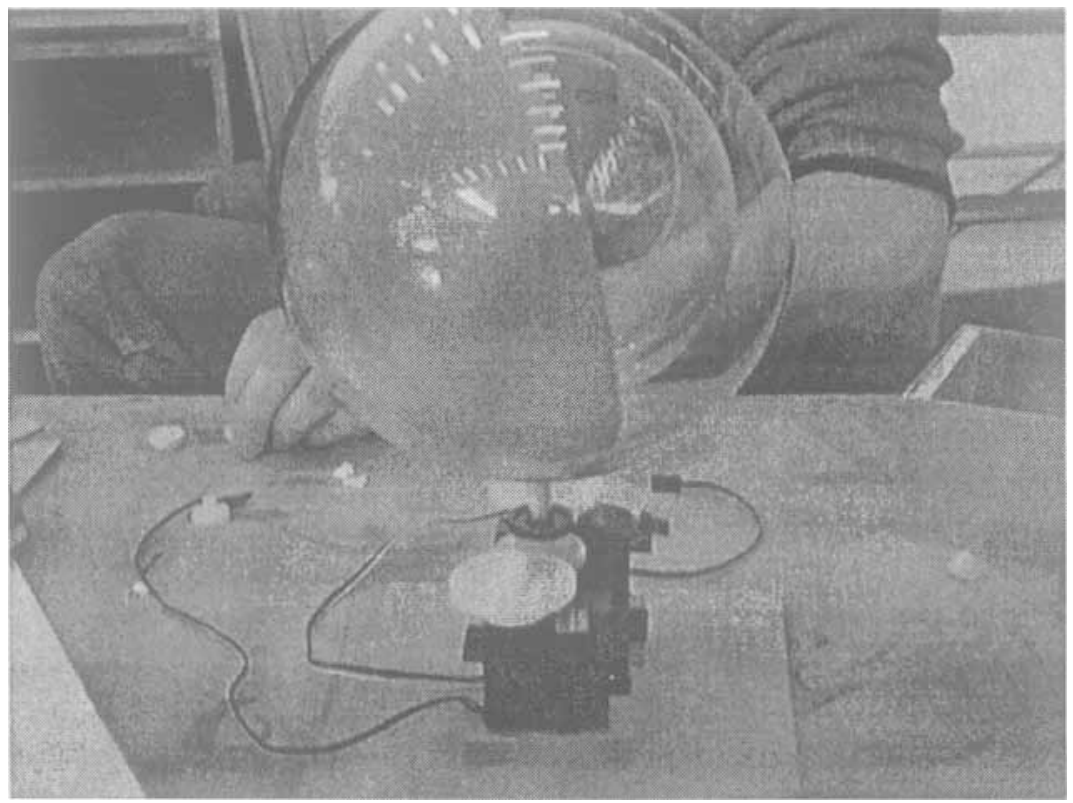

Figure 7. 4D sketching explorative(2)

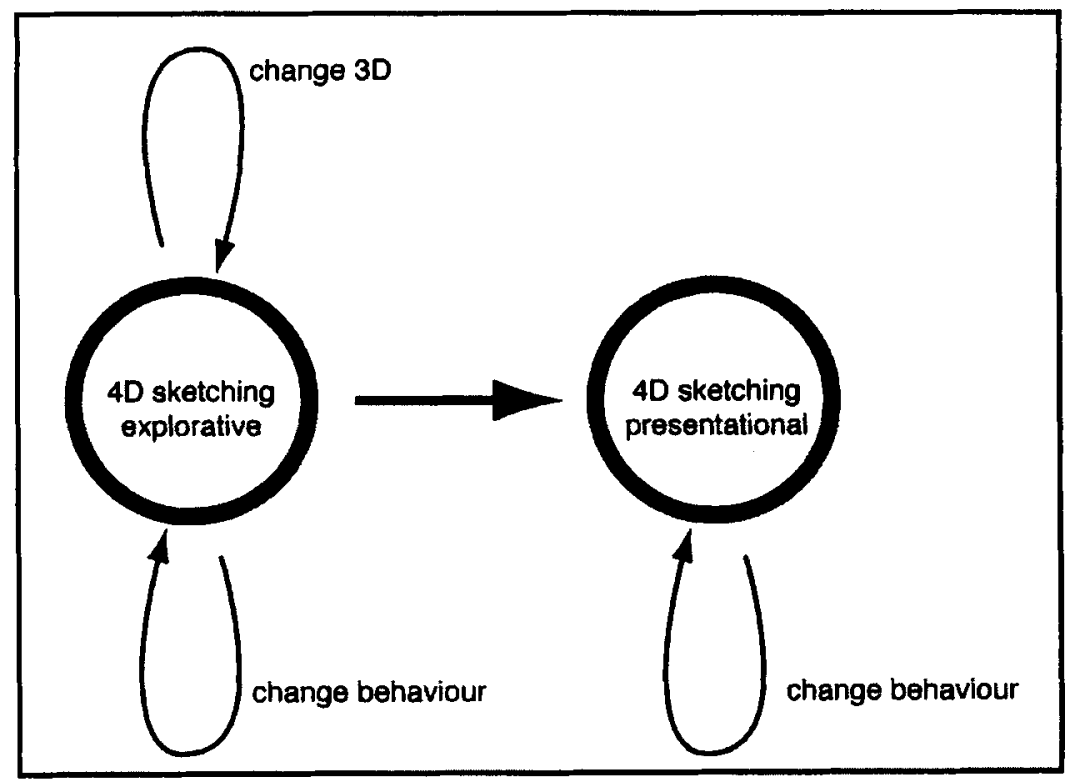

Figure 8. 4D sketching explorative(3) 


\section{EXAMPLES AND EXPERIENCES}

\subsection{Phone cradles}

The 4D sketching technique has been used in the (ongoing) telephone cradle project to explore whether an active cradle can express emotions. The 4D sketch of Figure 9 is quite capable of expressing "joy" through wobbling (it is kind of top-heavy and therefore the base wobbles too).

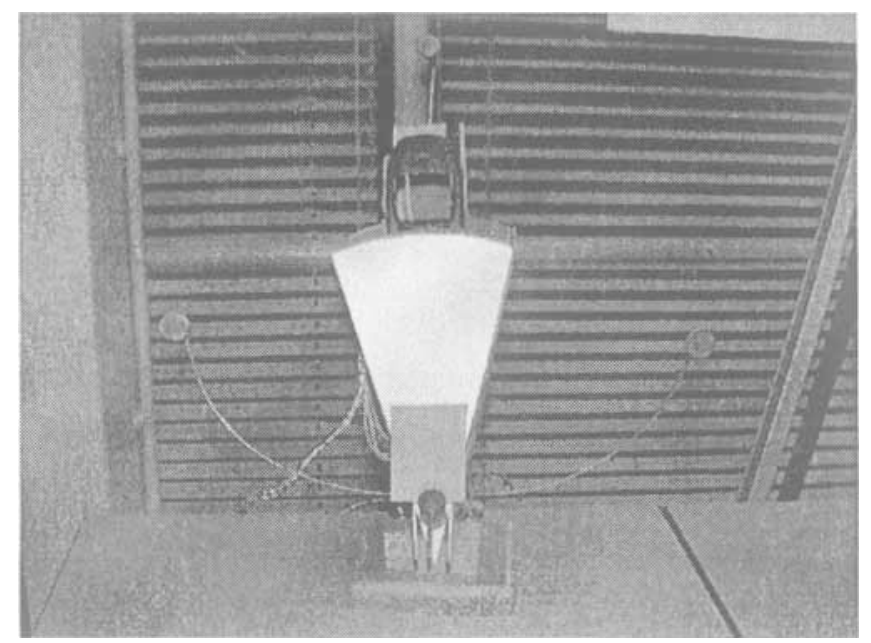

Figure 9. Active telephone cradle with wobble movement.

The models of Figures 9 and 10 have also been used to explore interaction. The model of Figure 9 has four touch-sensors. In each case the phone will move towards the user touching a sensor. Each the cradle has a lift; so when in Figure 6 the upper touch sensor is touched, the phone comes out whereas when the lower touch sensor is touched the phone moves back inside the cradle. The other model, shown in Figure 7, has just one sensor for the lift instead of two; the question we explored was whether users would recognize the "logic" of this second model, where the sensor works as a toggle switch (most test subjects had no difficulty with guessing or grasping the toggle behaviour). But it also became clear that involving test subjects demands a clear context or useful application.

Therefore in the student projects, clear choices for the context were made by us from the very beginning. The object was to perform a simple but useful function: to offer a little food. Then the students decided that this was to be a walnut. 


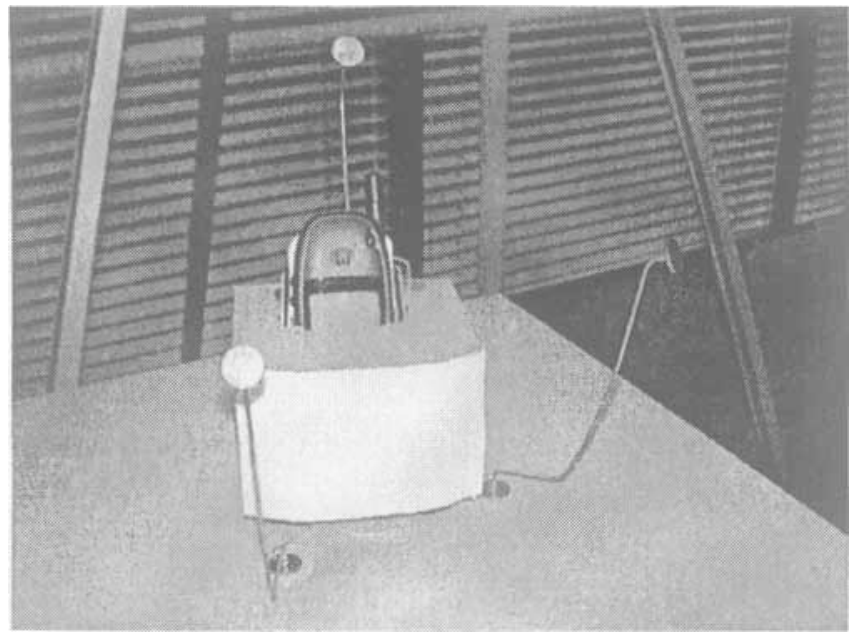

Figure 10. Active telephone cradle with twist movement

Here we describe the design rationale and the characteristics of the five walnut machines.

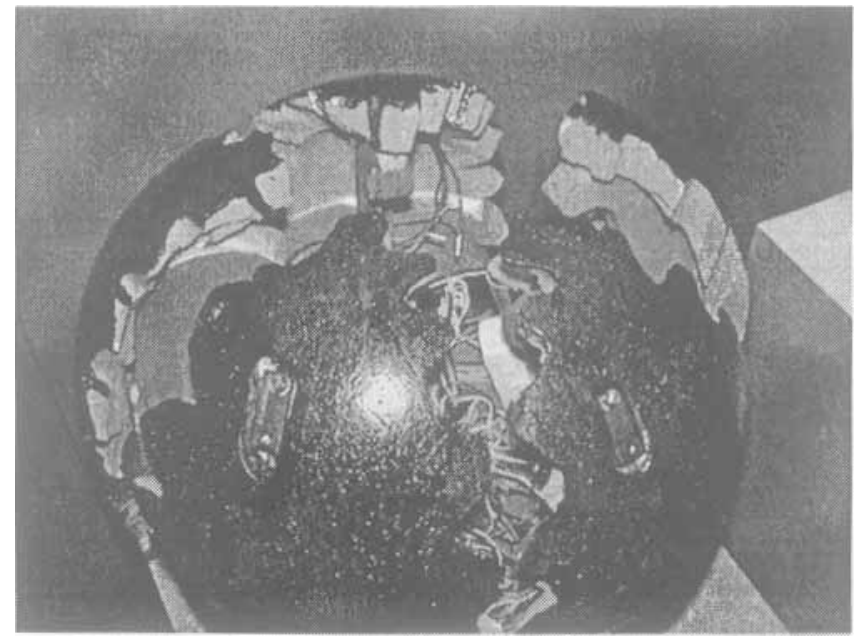

Figure 11. Crack, the angry walnut machine (closed)

\subsection{Crack (anger) -design: Rombout Frieling}

Frieling describes anger as a contrast between inside and outside, of initially cropped up inner tension against the outside world. Anger may change one's 
a trend of escalation. At the climax of one's anger, there may be a kind of anger overflow.

Frieling translated this description into 'Crack', a spherical machine whose shell consists of five blades with serrated edges. Initially, Crack's shell is fully closed with only the blood red, serrated spaces between its blades indicating its unrest. When approached, Crack lashes out at its user through increasingly large movements of one randomly chosen blade at a time. Finally, Crack totally cracks open into an aggressive angular, bloodred shape and ejects the walnut vertically.

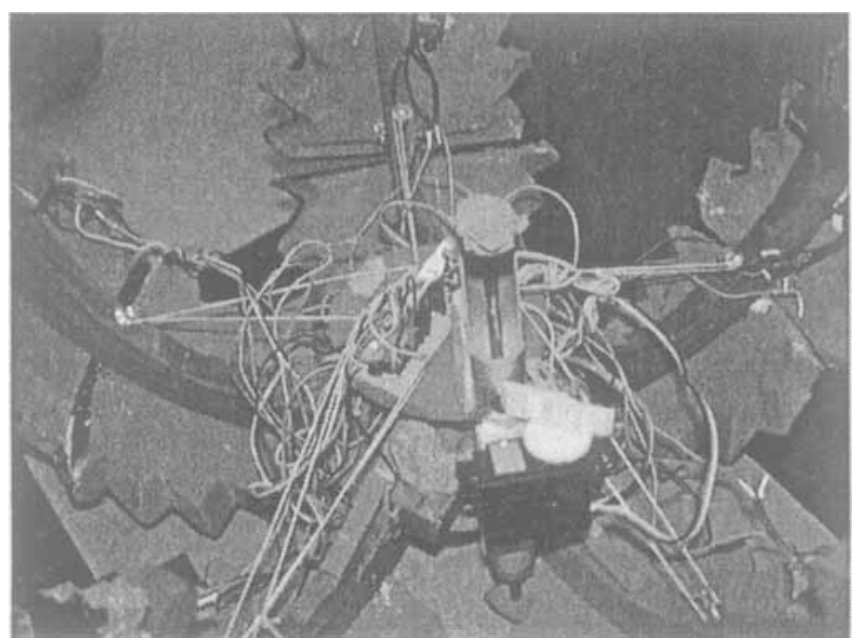

Figure 12. Crack, the angry walnut machine (open).

\subsection{Dolly (love) - design: Jan Hoefnagels}

In showing love or affection, one can do anything from comforting, via playing with, to teasing the loved one. Hoefnagels translated love into a machine called Dolly which has a wooden, smoothly polished, organic body. Dolly exhibits smooth and slow movements, intended to resemble a comforting caress. The movements curve and turn and topple past the user, giving an impression of teasing, of playing with or nuzzling up to him or her. Finally, eight bead-terminated pins slowly expand from Dolly's main body, offering the user the walnut. 


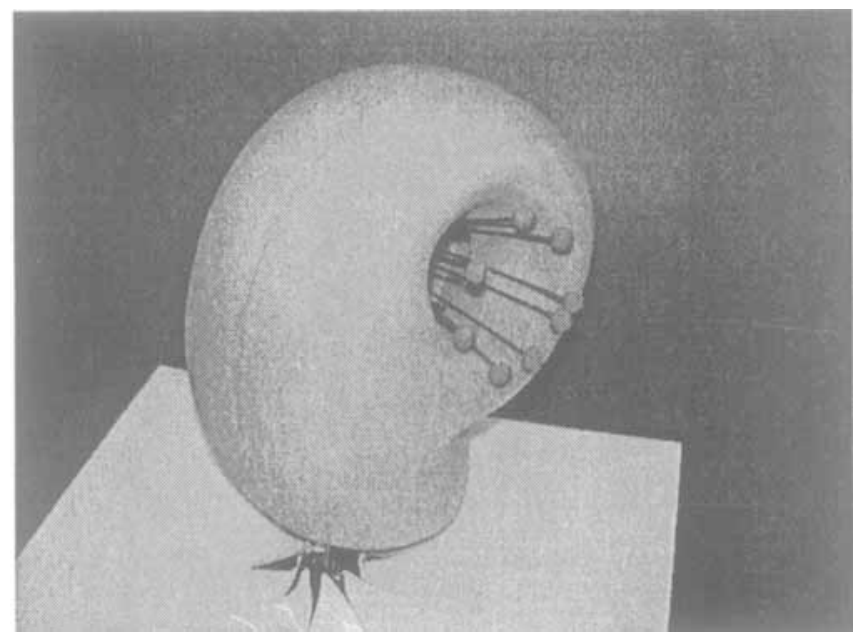

Figure 13. Dolly, the walnut machine in-love (nut still inside)

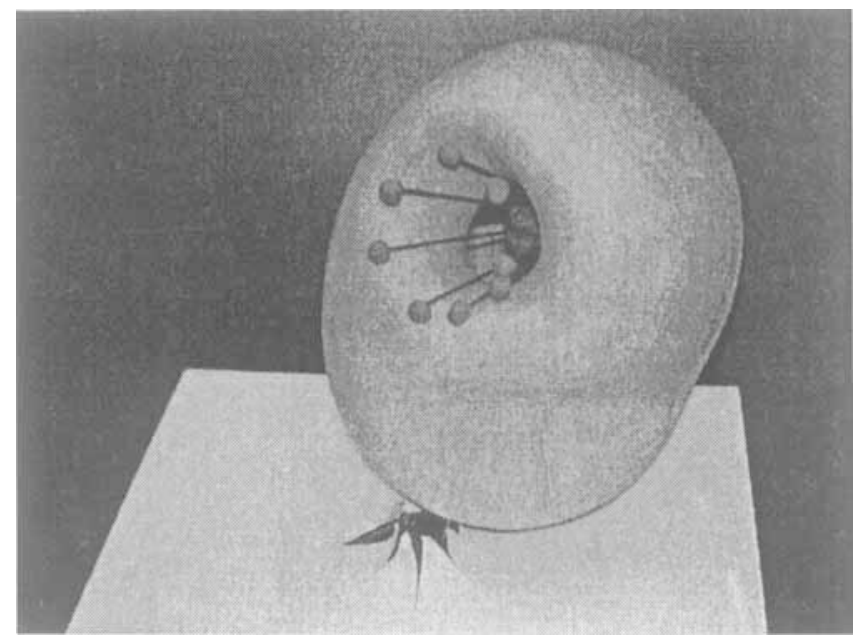

Figure 14. Dolly, the walnut machine in love (nut coming out)

\subsection{More Walnut machines}

Emotion: Enthusiasm. Ralph Zoontjes' model is used to explore expressing the emotion enthusiasm. His description of this emotion contains several stages. First, there is a suspense stage in which one expects an event that would be very advantageous. Second, one becomes more active and interested, concentrating on whether the event is going to happen or not. 
Then, as it is very likely for the event to happen, the excitement builds up, one start moving more, thus letting out some of the energy that has gathered inside. Finally, the real enthusiasm bursts out. From various observations (people watching football matches, happy dogs), Zoontjes concluded that there are many movement with high frequency and low amplitude to begin with but that enthusiasm often ends with very intense, large movements. Zoontjes implemented these ideas into Phunx, a bright red and green coloured machine of organic form. Initially, Phunx slowly 'wags' its red top. When approached, it starts wagging faster. Finally, it lifts its white 'walnut scoop' in three quick, increasingly large movements, leading to the walnut being launched in the user's direction.

Emotion: Panic. Dirk Volman noted the following dynamic characteristics of panic. Firstly, one's movements spring from a single point. Secondly, the movements lack focus, because one wants to do things all at once, worsening one's panic only further. And thirdly, a movement creates a counter-movement because one wants to do everything at once and because one doubts the initial move was effective. And each counter movement creates a counter movement which creates a counter movement etc. etc. Finally, one startles again as one realizes one's problems are solved after all. Volman translated these characteristics into a semi-spherical machine called Caos. Caos violently moves random sections of its shell outwards and retracts them again, to finally extend all of them simultaneously. The nut rolls out in a rather uncontrolled manner and, after a short break, Caos retracts all its moving parts and comes to rest.

Emotion: Shyness. Melika El-Massoudi noted that shy people first turn away from the requesting user, often shielding their face with one hand, blushing and hesitating between doing nothing and fulfilling the request. Her machine consists of two transparent semi-spherical plastic shells in whose center the walnut lies. As the user approaches the machine, the shells turn their back on the user, showing a different colour. They then move between shielding and exposing the walnut to, finally, fully expose it to the user.

\subsection{General observations}

On the basis of the given examples and experiences, a number of general observations can be made. First, it is absolutely worthwhile to create models which exist in the physical dimensions of the real world, not just 3D simulated in Virtual Reality. The point is that the interaction takes place by the user's real body in a multi-modal way. The sensors are not abstract buttons: the user really feels the touch button and the user notes that the IR 
sensors, depending on their type, do or do not have a directional sensitivity. Moreover, the sensors may move themselves relative to the phone or the nut.

Secondly, it became clear that form, colour, motion and even sound are hard to separate. In Crack and Caos these were designed to re-enforce each other (the snapping sound of solenoids fits well with panic); the surpriseeffect this had on test subjects was enormous.

Thirdly, we found that a well-defined context was indispensable when entering a rational dialogue with a user or a test-subject. On the other hand, even without proper context, observers were intrigued by any interaction behaviour or autonomous behaviour of the models, except for simple and predictable behaviour.

Finally, we found that there are basic laws of object motion that emerge during 4D sketching but that are easily overlooked when working in 2 or 3D. In particular, any finite translation or any rotation of less than $360^{\circ}$ has sooner or later to be followed by a movement in the opposite direction. For example, the blades of Crack move outward very fast, as a part of its expression of anger; but when folding-in gently, they weaken the anger. But closing fast again makes the expansion and the contraction symmetric, which is also not the designer's intention (for anger one needs the aggressive expansion). This type of problem continues to exist when there are several parallel, independent finite translations or limited rotations (like the four blades). But it is possible to find ways out of this dilemma when two movements are coupled (like the rotation and translation of Dolly).

\section{RELATED WORK}

In recent years there has been a growing interest in toolkits which facilitate the building of physical, mechatronic prototypes, such as Phidgets (Greenberg \& Boyle, 2002), iStuff (Ballagas et al., 2003) and the Calder Toolkit (Lee et al., 2004). What these toolkits have in common is that they intend to make the creation of electronic prototypes possible for non-experts, i.e. those not skilled in electronic engineering or information science. Frequently, an analogy is drawn with graphical user interfaces in which GUI toolkits with drag and drop widgets that come with their own behaviour have made possible the development of GUI applications by non-programmers. All of these toolkits make use of physical input and output building blocks that can be linked to an application running on a PC. The main idea behind this approach is building on existing knowledge: by using a PC the user can program behaviour in a graphical environment using high level languages such as Macromedia Director's Lingo, Visual Basic or Java that many in the interaction design community are already familiar with. 
In contrast with these toolkits, our 4D sketching method does not rely on such tight integration between electronics hardware and the PC. The hardware components are not represented in anyway in the graphical environment and do not require drivers. Instead, the PC is merely used to type in JAL code and download it to PIC. We deliberately do not use a PIC simulator' on the PC: all exploration is done through rapid iteration cycles, comparing the behaviour in the physical world with the designer's expectations. Actually, the exploration process starts at the beginning of the project when a working $\mathrm{I} / \mathrm{O}$ program together with a sensor and servo is delivered to the students. From that point on, the process is a matter of modification, re-use and thus exploration. The combination of the JAL language and the PIC microcontroller offers direct control over input and output ports in one or two lines of code. The JAL language is easy to step into and has a gradual learning curve, just like Basic. Typically, JAL leads to small, single file programs that are easy to read and encourage re-use of software.

Compared to other microcontrollers with easy to learn languages such as the Parallax BasicStamp and Javelin, the PIC microncontroller is very cheap. The high spec 168877 that we use here costs around 10 USD. A comparable BasicStamp or Javelin costs about five to nine times as much. The low cost of the PIC not only makes it less costly to create multiple prototypes, it also means that we can experiment more freely: a couple of burned microcontrollers won't break the bank.

Finally, Microchip, the makers of the PIC microcontroller, offer a path for further miniaturization. Whilst our programming board makes use of relatively large 40 pin DIL packages, the JAL code will run 'as is' using the same input/output pins on a miniature surface mount version of the same controller.

\section{EVALUATION AND OUTLOOK}

We described how we and our students made the leap towards sketching in the temporal dimension. As we are based at a technical university, we are interested in letting students gain proficiency in integrating technology in a practical, hands-on manner, thus creating a new designer 'breed' that can move beyond static models and on-screen simulations towards working, mechatronic prototypes.

It was possible to "package" the $4 \mathrm{D}$ sketching approach by instructing the students on the acting-out techniques (Sect. 3.4) and by providing them with the materials shown in Figures 4 and 5 as a part of a two-our demo/instruction session (the default was two servos, a PIC and a sample 
program; other actuators and sensors were dependent on each student's own initiative). Once the technicalities of $4 \mathrm{D}$ sketching are mastered, creating a new model from scratch takes less than four hours. Modifying an existing model for exploration purposes is a matter of minutes.

Let us return to the question from the introduction: How can the form, empowered with behaviour, communicate human values, culture values, societal values, tribal, community, brand values? It has become clear that objects can express at least basic emotions through movement. The 4D sketching technique is useful as a tool to explore basic emotional expressions and at least promising to explore the next levels of complexity, mediating values.

\section{ACKNOWLEDGMENTS}

We gratefully acknowledge the enthusiastic contributions of our students Rombout Frieling, Jan Hoefnagels, Raplph Zoontjes, Dirk Volman, and Melika El-Massoudi, the technical support of Ton van der Graft and the help and cooperation offered by Kees Overbeeke and Joep Frens.

\section{REFERENCES}

Aldersey-Williams, H., Wild, L. Boles, D., McCoy, K., McCoy, M., Slade, R., \& Diffrient, N. (1990). The New Cranbrook Design Discourse. New York: Rizzoli International Publications.

Avrahami, D., \& Hudson, S.E. (2002) Forming interactivity: a tool for rapid prototyping of physical interactive products. Proceedings of DIS2002, London, pp. 141-146Ballagas, R., Ringel, M., Stone, M., \& Borchers, J. (2003). iStuff: a Physical User Interface Toolkit for Ubiquitous Computing Environments. Proceedings of CHI2003, pp. 537-544.

Burns, C; Dishman, E; Verplank, W. and Lassiter, B, (1994) Actors,Hairdos \&Videotape Informance Design. In: $\mathrm{CHI}$ ' 94 Conference Companion, ACM, Boston.

Greenberg, S., \& Boyle, M. (2002). Customizable physical interfaces for interacting with conventional applications. Video Proceedings of the ACM UIST 2002 15th Annual ACM Symposium on User Interface Software and Technology. ACM Press.

Hummels, C.C.M., Djajadiningrat, J.P., \& Overbeeke, C.J. (2001). Knowing, doing and feeling: communicating with your digital products. Interdisziplinăres Kolleg Kognitionsund Neurowissenschaften, Günne am Möhnesee, March 2-9 2001, 289-308.

JAL open source project: https://sourceforge.net/projects/jal/

Lee, J.C., Avrahami, D., Hudson, S.E., Forlizzi, J., Dietz, P.H., \& Leigh, D. (2004). To appear in the proceedings of DIS2004.

Krippendorff, K., \& Butter, R. (1984). Product semantics: Exploring the symbolic qualities of form. Innovation. The Journal of the Industrial Designers Society of America, pp.4-9. 